Original Research Article

\title{
A prospective study comparing the safety and efficacy of combination of aceclofenac and thiocolchicoside against aceclofenac alone in low back pain
}

\author{
Aparna Mathummal Nellika ${ }^{1 *}$, Anuradha Mothalampet ${ }^{1}$, Sooraj Rajagopal ${ }^{2}$
}

\begin{abstract}
${ }^{1}$ Department of Pharmacology, ${ }^{2}$ Department of Physical Medicine and Rehabilitation, Government Medical College, Kozhikode, Kerala, India
\end{abstract}

Received: 27 October 2017 Accepted: 24 November 2017

\section{*Correspondence to:}

Dr. Aparna Mathummal Nellika, Email: draparnamn@gmail.com

Copyright: (C) the author(s), publisher and licensee Medip Academy. This is an openaccess article distributed under the terms of the Creative Commons Attribution NonCommercial License, which permits unrestricted noncommercial use, distribution, and reproduction in any medium, provided the original work is properly cited.

\begin{abstract}
Background: Aceclofenac is a non steroidal anti-inflammatory drug commonly prescribed in patients with acute low back pain. Thiocolchicoside is a skeletal muscle relaxant which is used in combination with NSAIDs. The efficacy of a combination of aceclofenac and thiocolchicoside has to be proved over aceclofenac alone in patients with acute low back pain. Objective of this study was to compare the safety and efficacy of a combination of aceclofenac and thiocolchicoside against aceclofenac alone in patients with acute low back pain. Methods: This study was undertaken as a prospective comparative study. Patients with acute low back pain receiving either aceclofenac $100 \mathrm{mg}$ or a combination of aceclofenac $100 \mathrm{mg}$ and thiocolchicoside $4 \mathrm{mg}$ twice daily were enrolled in the study and were divided into two groups of 50 each. The primary efficacy parameter was pain intensity measured on a visual analogue scale. Adverse effects if any were monitored at the follow up visit.

Results: At the start of the study, pain intensity, measured on visual analogue scale was comparable in both the groups. At the end point, there was a reduction in pain intensity in both the groups and the reduction was more significant in the combination group ( $\mathrm{p}<0.001)$. Adverse effects reported in both the groups were found to be comparable.

Conclusions: Combination of aceclofenac and thiocolchicoside is superior to aceclofenac alone in patients with acute low back pain.
\end{abstract}

Keywords: Aceclofenac, Low back pain, Thiocolchicoside, Visual Analogue Scale

\section{INTRODUCTION}

Low back pain has become a major health and socioeconomic problem and a leading cause of disability and loss of productivity. It is experienced by most of the population at some point of time in their lives. Studies have shown lifetime prevalence as high as $84 \%$ and about $40 \%$ of people say they have had low back pain within the past six months. ${ }^{1}$
The occurrence of low back pain is almost similar in all cultures and is a major cause of disability affecting performance at work and general well-being. Low back pain can be acute, sub-acute, or chronic. ${ }^{2}$ Even though, a vast array of risk factors has been identified, the causes of the onset of low back pain remain obscure.

Despite the availability of several guidelines for diagnostic and therapeutic approaches, early and effective analgesia 
combined with various mechanisms to enhance efficacy and reduce toxicity is the most suitable approach. ${ }^{3,4}$

Medications commonly used for the treatment of acute low back pain include non steroidal anti-inflammatory drugs, muscle relaxants, opioid analgesics, anti-depressants and systemic steroids either used alone or in combination.

Other alternative treatments include physical modalities, rehabilitation measures and spinal manipulation. Surgery is often the last option, when all other strategies have failed, and the outcomes can sometimes, be disappointing. So there has been a recent shift of focus more towards conservative care rather than surgical treatment.

Aceclofenac is a non steroidal anti inflammatory drug, indicated for symptomatic treatment of pain and inflammation. It is available as oral and topical formulations. Nausea, dyspepsia, diarrhoea, flatulence, constipation are the usual side effects. ${ }^{5}$ Thiocolchicoside is a semi synthetic colchicoside, obtained from the seeds of the plant Gloriosa superba. It is a centrally acting skeletal muscle relaxant with GABA mimetic and glycinergic actions. Its muscle relaxant effects could be exerted by complex regulatory mechanisms at the supraspinal level. It is indicated for the treatment of painful muscle spasms due to musculo skeletal disorders. ${ }^{6}$ Adverse effects include sedation, drowsiness, nervousness, photosensitivity, dyspepsia, insomnia and weakness. Rarely, seizures and liver injury have occurred.

Combination of Aceclofenac and Thiocolchicoside may maximize pain relief by different mechanisms. Though there is much controversy about the role of muscle spasm in acute back pain, there is no doubt that in extremely painful conditions, the use of a muscle relaxing drug combined with analgesics may prove to be a useful measure. , $^{8}$

\section{METHODS}

The study was designed as a prospective comparative study. It was conducted in the Department of Physical Medicine and Rehabilitation, Government Medical College, Kozhikode and Department of Pharmacology, Government Medical College, Kozhikode after getting clearance from the Institutional Research Committee and Institutional Ethics Committee of Government Medical College Kozhikode. It was conducted over a period of one year from January 2014 to January 2015. Written informed consent was obtained from the patients in vernacular language before starting the study.

Patients in the age group of 18 to 65 years with localized uncomplicated low back pain of recent onset (less than 3 months) with a pain intensity of at least 6 on a 10 point Visual Analogue Scale were included in the study. Patients with known hypersensitivity to the study drugs, those with history of malignancy, osteoporosis, previous spinal surgeries, abnormal liver or renal function tests and those with history of seizure disorders were excluded from the study.

After screening, patients who fulfilled the inclusion criteria were enrolled in the study. A total of 100 patients were enrolled in the study. Patients were divided into two groups - Group I and Group II of fifty each.

Group I included patients receiving aceclofenac 100mg twice daily and Group II included patients receiving a combination of aceclofenac 100mg and thiocolchicoside 4 $\mathrm{mg}$ twice daily for seven days. Cap Omeprazole $20 \mathrm{mg}$ was added as a rescue medication in both the groups.

Baseline parameters of the patients were recorded. Intensity of pain was assessed on day 0 using visual analogue scale. Visual Analogue Scale is a horizontal line $10 \mathrm{cms}$ in length. A score of 0 represents "no pain" and a score of 10 the "worst possible pain" the patient has experienced. The patients were asked to mark a point on the line that they feel which represents their perception of pain in the present state. The measurements were taken in millimetres from the left-hand end of the line to the point the patient has marked. Patients were asked to report after seven days and the final pain intensity was measured again using visual analogue scale. Outcome was assessed by measuring the decrease in pain intensity Spinal range of motion was assessed on day 0 and day 7 by clinical assessment.

Restriction of range of motion was graded as follows:

- $\quad 0$ - Full range of motion possible

- $\quad 1$ - Upto $25 \%$ restricted

- $\quad 2-25-50 \%$ restricted

- $3->50 \%$ restricted

Adverse drug reactions were monitored during the study period based on history, observation and self reported adverse drug reactions. Physical examination was done during both the visits. Patients were asked to report immediately if they experienced any adverse effects and they were recorded. 48 patients in group 1 and 49 patients in group 11 completed the study.

\section{RESULTS}

Statistical analysis was done using Statistical Package for Social Science (SPSS) software. All patients who completed the treatment were included in the statistical analysis. Gender distributions of the two groups were compared using Chi square test. Baseline parameters like age and BMI were compared using independent samples $\mathrm{t}$ test. VAS scores were also compared using independent samples t test. Spinal range of motion was compared using Chi square test. Adverse effects in both the groups were compared using Chi square test. $\mathrm{p}$ value of less than 0.05 was considered statistically significant. 
Out of the 97 patients who completed the study, $56.7 \%$ were females and $43.3 \%$ were males.

Age is considered to be a common risk factor for low back pain. Several studies have shown that the prevalence of low back pain increases with increasing age. In the present study, $53.6 \%$ of the patients were in the age group between 36 and 50 years and $27.8 \%$ in the age group between 51 and 65 years. The mean age in aceclofenac alone group was $46.3 \pm 10.63$ and in the combination group was $43.98 \pm 10.36$ and were found to be comparable $(p>0.05)$. The mean BMI in aceclofenac alone group was $24.36 \pm 2.90$ and in the combination group was $23.76 \pm 2.95$ ( $p>0.05$ ).

The primary efficacy parameter was pain intensity measured on a visual analogue scale. The baseline VAS scores of the patients were assessed on the first visit. The mean VAS score for aceclofenac group was $7.077 \pm 0.89$ and in the combination group, it was $7.273 \pm 0.92$. The baseline scores were compared using independent samples $\mathrm{t}$ test and were found to be comparable ( $\mathrm{p}>0.05)$. Range of spinal flexion and rotation was also assessed on both the visits.

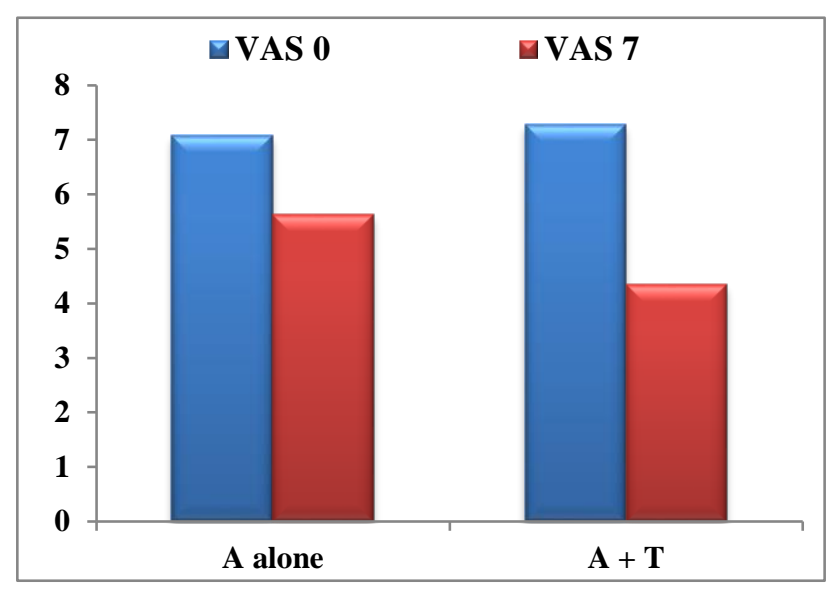

\section{Figure 1: Comparison of baseline and final vas score in the study groups.}

The final VAS score in aceclofenac alone group was $5.63 \pm 1.18$ and in the combination group it was $4.35 \pm 1.79$. There was a significant reduction in the VAS scores in both the groups and the reduction was more in the combination group. Statistical analysis was done using independent sample $t$ test and the reduction in VAS scores was found to be significantly better in the combination group compared to aceclofenac alone group $(p<0.001)$. There was also a significant improvement in the spinal flexion and rotation in the combination group compared to aceclofenac alone group. Statistical analysis was done using Chi-square test $(\mathrm{p}<0.05)$. The adverse effects reported in both the groups were mild. No serious adverse drug reactions occurred during the study and none of the adverse effects needed discontinuation of the drugs.

In the study, $10.4 \%$ of the patients in the aceclofenac alone group and $18.4 \%$ of the patients in the combination group reported adverse effects. Adverse effects reported in the aceclofenac alone group were nausea, dyspepsia and epigastric pain and in the combination group were nausea, dyspepsia, giddiness and diarrhoea. Clinically, the adverse effects reported in the combination group were more compared to aceclofenac alone group. Statistical analysis was done using Chi square test and the difference was not found to be statistically significant $(\mathrm{p}>0.05)$.

\section{DISCUSSION}

Low back pain is an extremely common health problem and a leading cause of activity limitation and work absence throughout the world. It is a symptom, not a disease and has a variety of causes. Most of the patients have only short attacks of pain of mild to moderate intensity but they tend to recur over many years. Until a few years ago it was largely thought of as a problem confined to the Western world but increasing researches in the field have shown that it has become a major problem in the middle and low income countries. There has been a steady increase in the percentage of patients disabled by back pain and it has become a costly burden to the society as well.

Most of the studies on various treatments of low back pain have unfortunately shown limited efficacy, especially in chronic back pain. The most commonly prescribed treatments such as medications, exercise and spinal manipulation tend to show only mild to moderate improvement in large trials and most of the physicians use multiple treatments on patients in the hope that their cumulative effect will provide sufficient pain relief and an improvement in symptoms. ${ }^{1}$

Many studies have provided strong evidence that non steroidal anti inflammatory drugs provide pain relief in both acute and chronic low back pain. NSAIDs provide pain relief by inhibiting cyclooxygenase enzyme and thus decreasing $\mathrm{PGE}_{2}$ synthesis.

Thiocolchicoside, a semi synthetic derivative of colchicine, is a commonly used muscle relaxant for the treatment of acute muscle spasms. It has additional anti inflammatory and analgesic properties.

Several studies have shown that FDCs of non steroidal anti inflammatory drugs and skeletal muscle relaxants are superior in efficacy in the treatment of acute low back pain compared to the former being used alone. The results of the present study suggest that a combination of aceclofenac and thiocolchicoside is superior in efficacy compared to aceclofenac alone.

The study was conducted in a single centre in a homogenous group of patients. It was not randomized or blinded. The primary efficacy parameter was intensity of pain expressed on a visual analogue scale which is a subjective measure and it is difficult to measure pain based on intensity alone. 


\section{ACKNOWLEDGEMENTS}

Authors would like to thank their guide Dr. Anuradha M., Professor and Head of the Department of Pharmacology, Govt. Medical College, Kozhikode, who with her able guidance and constant encouragement gave them all the confidence and determination to complete the study.

Authors also would like to express their deepest sense of gratitude and thanks to Dr. Sooraj Rajagopal, Associate Professor, Department of Physical Medicine and Rehabilitation Govt. Medical College Kozhikode, for his able guidance and support during the study.

Above all, authors sincerely thank God Almighty for enabling them to complete this study.

Funding: No funding sources

Conflict of interest: None declared

Ethical approval: The study was approved by the Institutional Ethics Committee

\section{REFERENCES}

1. Barr KP, Harrast MA. Low Back Pain. In: Randall L. Braddom. Physical Medicine and Rehabilitation. $4^{\text {th }}$ Ed. Philadelphia: Elsevier; 2011:871-905.

2. Ehrlich GE. Backpain. J Rheumatol suppl. PubMed. 2003 Aug;67:26-31.
3. Desmeules J, Rollanson V, Piguet V, Dayer P. Clinical pharmacology and rationale of analgesic combination. Eur j Anaesthesiol. 2003;28:7-11.

4. Raffa RB, Clark- Vetvi R, Tallarida RJ, Wertheimer AI. Combination strategies for pain management. Expert Opin Pharmacother. 2003 Oct;4(10):1697-768.

5. Dooley M, Spencer CM, Dunn CJ. Aceclofenec: a reappraisal of its use in the management of pain and rheumatic disease. Drugs. 2001;61(9):1351-78.

6. Patat A, Klein MJ, Surjus A. Effects of acute and repeated does of muscle relaxant chlormezanone and thiocolchicoside on vigilance and psychomotor performance of healthy volunteers. Human Psycopharmacology. 1991;6:285-92.

7. Cherkin DC, Wheeler KJ, Barlow W, Deyo RA. Medication use for low back pain in primary care. Spine. 1998;23(5):607-14.

8. Ressignol M, Abenhaim L, Bonvalot Y, Gobeille D, Shrier I. Should the gap be filled between guidelines and actual practice for management of low back pain in primary care? Spine. 1996;21:2893-8.

Cite this article as: Nellika AM, Mothalampet A, Rajagopal S. A prospective study comparing the safety and efficacy of combination of aceclofenac and thiocolchicoside against aceclofenac alone in low back pain. Int J Basic Clin Pharmacol 2018;7:333-6. 